\title{
Risk Factors of and Treatments for Pharyngocutaneous Fistula Occurring after Oropharynx and Hypopharynx Reconstruction
}

\author{
Su Bin Do ${ }^{1}$, Chul Hoon Chung ${ }^{1}$, Yong Joon Chang ${ }^{1}$, Byeong Jun $\mathrm{Kim}^{1}$, Young Soo Rho ${ }^{2}$ \\ Departments of ${ }^{1}$ Plastic and Reconstructive Surgery and ${ }^{2}$ Otorhinolaryngology-Head and Neck Surgery, Hallym University College of \\ Medicine, Seoul, Korea
}

\begin{abstract}
Background A pharyngocutaneous fistula is a common and difficult-to-manage complication after head and neck reconstruction. It can lead to serious complications such as flap failure, carotid artery rupture, and pharyngeal stricture, and may require additional surgery. Previous radiotherapy, a low serum albumin level, and a higher T stage have been proposed as contributing factors. We aimed to clarify the risk factors for pharyngocutaneous fistula in patients who underwent flap reconstruction and to describe our experiences in treating pharyngocutaneous fistula.

Methods Squamous cell carcinoma cases that underwent flap reconstruction after cancer resection from 1995 to 2013 were analyzed retrospectively. We investigated several significant clinical risk factors. The treatment modality was selected according to the size of the fistula and the state of the surrounding tissue, with options including conservative management, direct closure, flap surgery, and pharyngostoma formation.

Results A total of 127 cases (18 with fistulae) were analyzed. A higher T stage ( $P=0.048)$ and tube-type reconstruction $(P=0.007)$ increased fistula incidence; other factors did not show statistical significance $(P>0.05)$. Two cases were treated with conservative management, 1 case with direct closure, 4 cases with immediate reconstruction using a pectoralis major musculocutaneous flap, and 11 cases with direct closure (4 cases) or additional flap surgery (7 cases) after pharyngostoma formation.

Conclusions Pharyngocutaneous fistula requires global management from prevention to treatment. In cases of advanced-stage cancer and tube-type reconstruction, a more cautious approach should be employed. Once it occurs, an accurate diagnosis of the fistula and a thorough assessment of the surrounding tissue are necessary, and aggressive treatment should be implemented in order to ensure satisfactory long-term results.
\end{abstract}

Keywords Cutaneous fistula / Postoperative complications / Myocutaneous flap / Free tissue flaps
Correspondence: Chul Hoon Chung Department of Plastic and Reconstructive Surgery, Hallym University College of Medicine, 150 Seongan-ro, Gangdong-gu, Seoul 05355, Korea

Tel: +82-2-2224-2246

Fax: +82-2-448-0114

E-mail:c21ps@hanmail.net

This article was presented at the PRS KOREA 2016 on November 19, 2016 in Seoul, Korea.

\section{INTRODUCTION}

A pharyngocutaneous fistula is a communication between the digestive tract and skin of the neck. It is a common complication associated with flap reconstruction or primary closure of a pharyngeal defect following resection of a malignant neoplasm. Ac- 
cording to the literature, its incidence varies from $8 \%$ to $22 \%$ [1]. Most cases occur within 1-4 weeks after surgery. Breakdown of the mucosal closure can cause saliva to flow into nearby soft tissues, and wound healing may be delayed in such a saliva-soaked environment, which may result in chronic inflammation and hypoxia of the neck skin and vasculature, leading to formation of a fistula [2].

When a fistula forms, it increases the risk of wound infection, which can lead to flap failure, and inflammation that spreads to the nearby carotid artery and jugular vein may cause these vessels to rupture. It can also influence the overall treatment and performance status of patients. Oral diet initiation may be delayed, time-consuming surgery may be required depending on the fistula's severity and other accompanying complications, and the cancer recurrence rate may be increased because of delays in administering anticancer therapy such as radiotherapy. Moreover, a long-standing fistula causes extensive scar formation that may lead to permanent sequelae such as pharyngeal stricture. Once they occur, fistulae are a major cause of increased mortality and morbidity.

The risk factors can be categorized into patient-related factors (low serum albumin level $[1,3,4]$, low hemoglobin level [4-6], and other comorbidities such as diabetes mellitus $[1,3]$, chronic obstructive pulmonary disease [5], chronic hepatopathy [3], and cardiopathy [7]), disease-related factors (previous radiotherapy $[3,5,8,9]$, a supraglottic tumor subsite $[5,7]$, a higher $\mathrm{T}$ stage $[5,10,11]$, positive surgical margins $[5,12]$, neck dissection $[5,7]$, preoperative tracheostomy [6]), and reconstruction-related factors (hypopharyngeal reconstruction $[4,5]$, the type of flap used in reconstruction [13], and excessive tension at the anastomosis site $[5,14])$. However, few risk factor analyses have specifically been conducted of patients who underwent reconstruction with a flap.

The present study aimed to analyze the causative factors of pharyngocutaneous fistula through a retrospective analysis of cases involving reconstruction by a single surgeon at a single institute, as well as to describe our treatment experiences.

\section{METHODS}

The present study was a retrospective analysis of cases involving pharyngeal reconstruction with a pectoralis major musculocutaneous (PMMC) flap or cutaneous free flap following resection of squamous cell carcinoma of the oropharynx, hypopharynx, or larynx from 1995 to 2013 (Table 1). We preferred to use a cutaneous free flap, but the PMMC flap was used for reconstruction in patients with a poor general condition, incomplete tumor resection, or a vessel-depleted neck (Fig. 1). All patients
Table 1. Patient characteristics

\begin{tabular}{|c|c|}
\hline Variable & No. $(\%)$ \\
\hline \multicolumn{2}{|c|}{ Age, yr (mean, 63.0; range, 26-86) } \\
\hline$\geq 60$ & $83(65.4)$ \\
\hline$<60$ & $44(34.6)$ \\
\hline \multicolumn{2}{|l|}{ Sex } \\
\hline Male & $122(96.1)$ \\
\hline Female & $5(3.9)$ \\
\hline \multicolumn{2}{|l|}{ Previous treatment } \\
\hline Primary tumor & $113(89.0)$ \\
\hline Recurrent tumor & $14(11.0)$ \\
\hline \multicolumn{2}{|l|}{ Tumor site } \\
\hline Oropharynx & $11(8.7)$ \\
\hline Hypopharynx & $100(78.7)$ \\
\hline Larynx & 16 (12.6) \\
\hline \multicolumn{2}{|l|}{ T stage } \\
\hline $\mathrm{T} 1$ & $3(2.4)$ \\
\hline T2 & $46(36.2)$ \\
\hline T3 & $28(22.0)$ \\
\hline $\mathrm{T} 4$ & $50(39.4)$ \\
\hline \multicolumn{2}{|l|}{ Type of flap } \\
\hline PMMC & $21(16.5)$ \\
\hline Free flap & $106(83.5)$ \\
\hline \multicolumn{2}{|l|}{ Type of free flap } \\
\hline RFFF & $97(76.4)$ \\
\hline ALTFF & $9(7.1)$ \\
\hline \multicolumn{2}{|c|}{ Type of reconstruction } \\
\hline Tube-type & $17(13.4)$ \\
\hline Patch-type & $110(86.6)$ \\
\hline \multicolumn{2}{|c|}{ Type of reconstruction (free flaps only) } \\
\hline Tube-type & $17(13.4)$ \\
\hline Patch-type & $89(70.1)$ \\
\hline \multicolumn{2}{|l|}{ Size of the flap } \\
\hline$<200 \mathrm{~cm}^{2}$ & $42(33.1)$ \\
\hline $200-300 \mathrm{~cm}^{2}$ & $42(33.1)$ \\
\hline $300-400 \mathrm{~cm}^{2}$ & $30(23.6)$ \\
\hline $400-500 \mathrm{~cm}^{2}$ & $6(4.7)$ \\
\hline$>500 \mathrm{~cm}^{2}$ & $7(5.5)$ \\
\hline \multicolumn{2}{|c|}{ Previous radiotherapy } \\
\hline Yes & $114(89.8)$ \\
\hline No & 13 (10.2) \\
\hline \multicolumn{2}{|c|}{ Preoperative albumin level } \\
\hline$\geq 3.5 \mathrm{~g} / \mathrm{dL}$ & $107(84.3)$ \\
\hline$<3.5 \mathrm{~g} / \mathrm{dL}$ & $20(15.7)$ \\
\hline \multicolumn{2}{|c|}{ Preoperative hemoglobin level } \\
\hline$\geq 12.5 \mathrm{~g} / \mathrm{dL}$ & $113(89.0)$ \\
\hline$<12.5 \mathrm{~g} / \mathrm{dL}$ & $14(11.0)$ \\
\hline \multicolumn{2}{|l|}{ Diabetes mellitus } \\
\hline Yes & 25 (19.7) \\
\hline No & $102(80.3)$ \\
\hline \multicolumn{2}{|l|}{ Smoking } \\
\hline Yes & 119 (93.7) \\
\hline No & $8(6.3)$ \\
\hline
\end{tabular}

underwent cervical lymph node dissection, and a single surgeon performed flap insetting. Vicryl 3-0 sutures were used for 2-layer suturing. 
To examine the effects of various clinical factors, fistula incidence was analyzed to identify associations with primary or recurrent cancer, tumor site, $\mathrm{T}$ stage, the type of flap used in the reconstruction, the size of the flap, preoperative radiotherapy,

Fig. 1. Flap selection in pharyngeal reconstruction

PMMC, pectoralis major musculocutaneous.

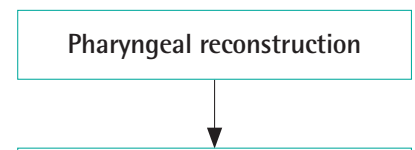

Poor general condition Incomplete tumor resection Vessel-depleted neck, etc.

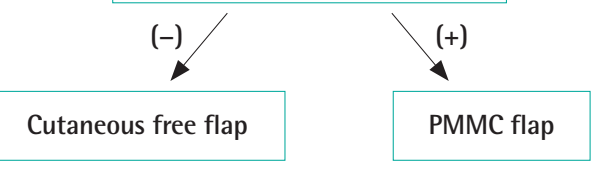

preoperative albumin level, preoperative hemoglobin level, diabetes mellitus status, and smoking status. SPSS ver. 23 (IBM Corp., Armonk, NY, USA) was used for the statistical analysis, and the Pearson chi-square test and a linear-by-linear association were used. P-values $<0.05$ were considered to indicate statistical significance.

When a pharyngocutaneous fistula occurs, the patient is immediately started on intravenous antibiotics and a compression dressing is applied, while avoiding oral feeding through a nasogastric tube or gastrostomy. Depending on the fistula size and the condition of the nearby tissues, an additional treatment modality is determined, with options including direct closure, flap surgery, and pharyngostoma formation. Direct closure was attempted when the fistula was small and the inflammation in the nearby tissues was mild, so that enough tissue remained after debridement around the fistula, whereas additional flap surgery was performed when the fistula was large or when direct closure was expected to cause functional disability. Moreover, when inflammation in the tissue around the fistula was severe and vascu-

Fig. 2. Patch-type PMMC flap for partial pharyngeal defect

A 56-year-old male with cancer of the hypopharynx. (A) Partial pharyngeal defect. (B) A PMMC flap was designed on the right anterior chest wall. (C) Insetting of the PMMC flap. The flap was transposed to the pharyngeal defect via a subcutaneous tunnel over the clavicle. (D) A video fluoroscopic swallowing study on postoperative day 16 showed a good pathway. PMMC, pectoralis major musculocutaneous.

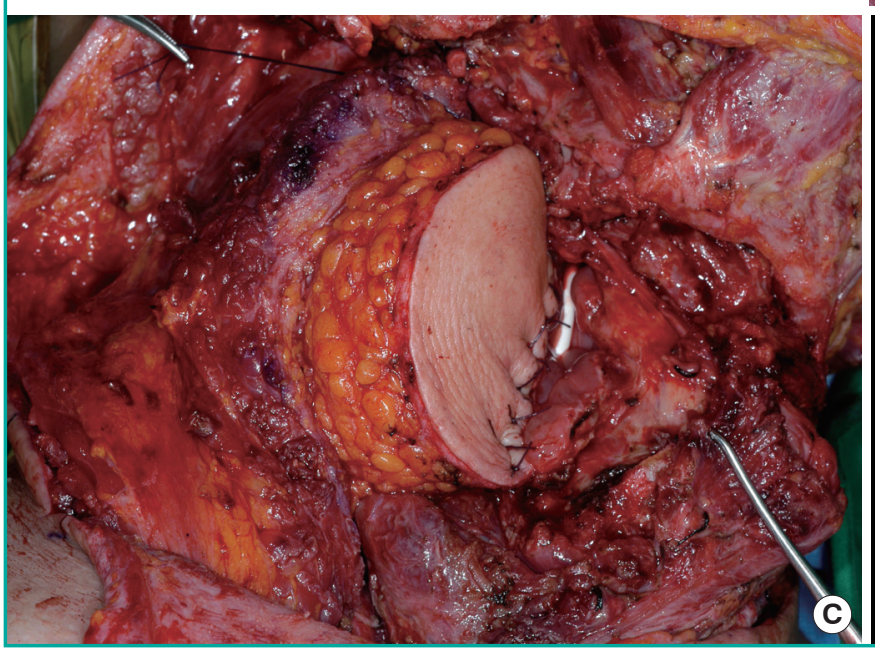

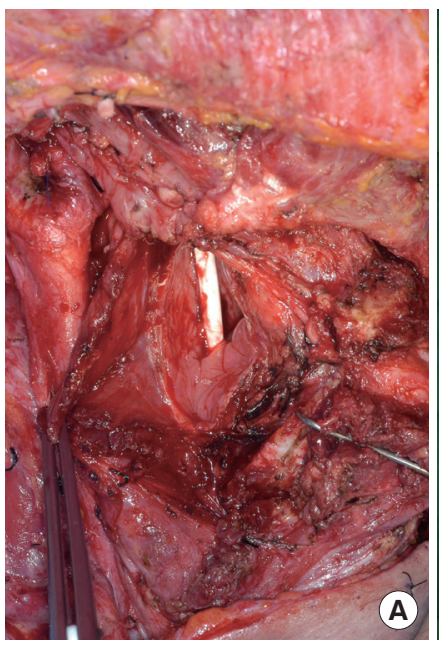
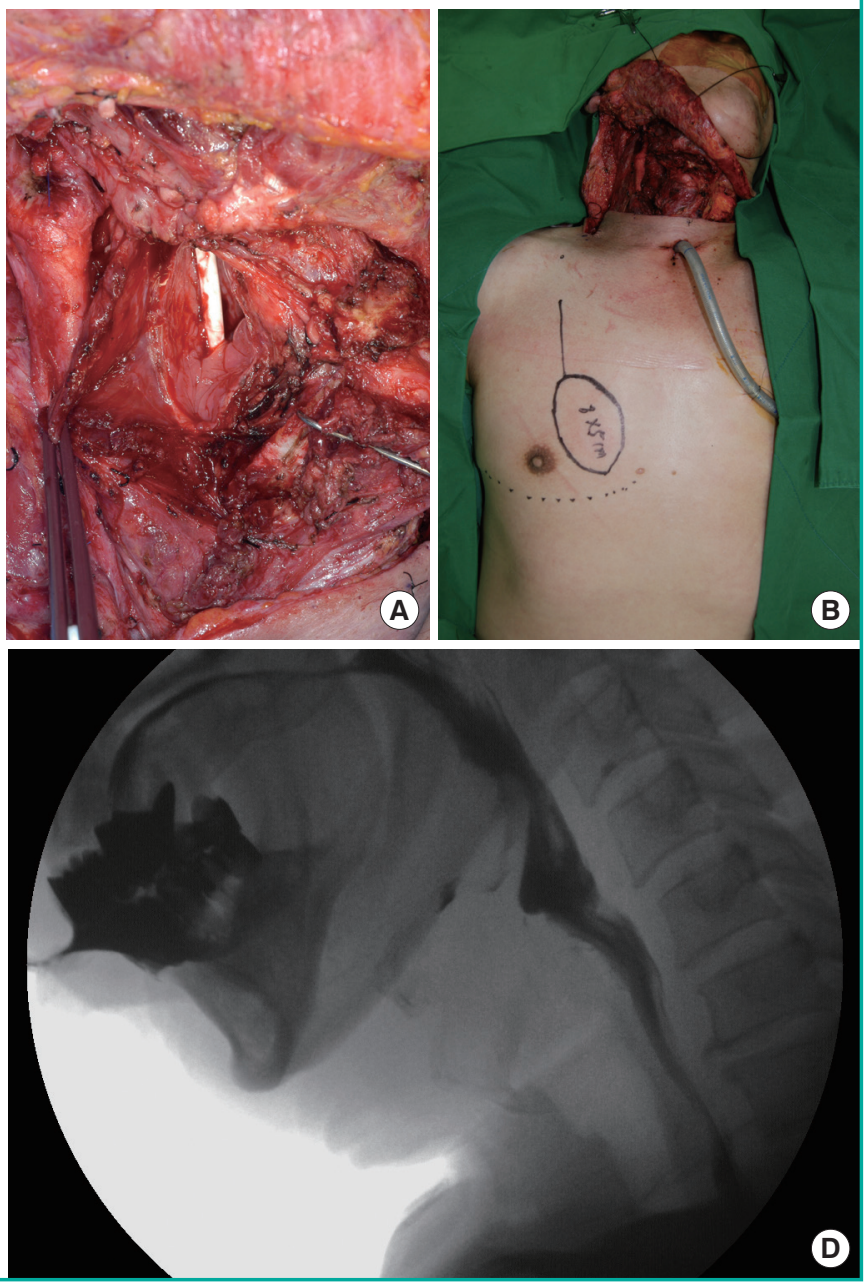
larity was suspected, pharyngostoma formation was performed to prevent saliva from directly contacting the wound, followed by pharyngostoma closure or reconstruction with a flap.

\section{RESULTS}

In total, 127 cases involving pharyngeal reconstruction with a PMMC flap (Fig. 2) or a cutaneous free flap following cancer resection were analyzed. There were 122 and 5 cases involving men and women, respectively; the mean age was 63.0 years (range, 26-86 years). A total of 21 cases were reconstructed using a PMMC flap, 106 cases using a cutaneous free flap, 97 cases using a radial forearm free flap, and 9 cases using an anterolateral thigh free flap. With respect to the different types of reconstructions, there were 17 and 110 cases of tube- (Fig. 3) and patchtype reconstructions, respectively. All PMMC flap reconstructions were patch-type. Therefore, within the free flap reconstruction group, excluding PMMC flaps, there were 17 and 89 cases of tube- and patch-type reconstructions, respectively.

Pharyngocutaneous fistula occurred in a total of 18 cases, resulting in an incidence of $14.2 \%$. Fistula incidence did not show a statistically significant association with primary or recurrent cancer, tumor site, size of the flap, preoperative radiotherapy, preoperative albumin level, preoperative hemoglobin level, diabetes mellitus status, or smoking status (Table 2). There were no differences in fistula incidence according to whether the oropharynx, hypopharynx, or larynx was the primary cancer site. Statistical significance could not be determined in the comparison of subsites because of an insufficient number of cases. Assessing the statistical significance of differences between the
PMMC and free flap reconstruction groups for the oropharynx, hypopharynx, and larynx sites was also impossible because of an insufficient number of cases.

Fistula incidence increased significantly with higher $\mathrm{T}$ stages $(P=0.015)$. With respect to flap types, there was no difference in fistula incidence between the PMMC and free flap reconstruction groups. A comparison between radial forearm and anterolateral thigh free flaps within the free flap reconstruction group was also impossible because of an insufficient number of cases. With respect to reconstruction types, a comparison between tube- and patch-type reconstructions showed that fistula incidence was significantly higher with a tube-type reconstruction, which was statistically significant when compared with the combined PMMC and free flap groups $(\mathrm{P}=0.006)$ and with the free flap reconstruction group excluding PMMC flaps $(\mathrm{P}=$ $0.007)$. In the 17 cases of tube-type reconstruction, a fistula occurred in 6 cases. In 4 cases, a fistula occurred between the base of the tongue and the flap, and in 2 cases a fistula occurred between the esophagus and the flap.

Fistulae were discovered between 6 and 27 days after surgery, at an average of 12 days. There were 2 cases in which leakage was discovered on an esophagogram obtained before initiating oral feeding in asymptomatic patients. They were treated with conservative management, but pharyngeal stricture occurred in both patients; subsequently, balloon dilatation was performed 65 days after surgery in a patient with cancer of the hypopharynx who underwent reconstruction with a tube-type radial forearm free flap and 63 months after surgery in a patient with cancer of the larynx who underwent reconstruction with a PMMC flap. In a single case, a patient with cancer of the hypopharynx

Fig. 3. Tube-type ALTFF for circumferential pharyngeal defect

A 72-year-old male with cancer of the hypopharynx. (A) Circumferential pharyngeal defect. (B) A tube-type ALTFF. (C) Insetting of a tube-type ALTFF. (D) Esophagography on postoperative day 16 showed a good pathway. ALTFF, anterolateral thigh free flap.
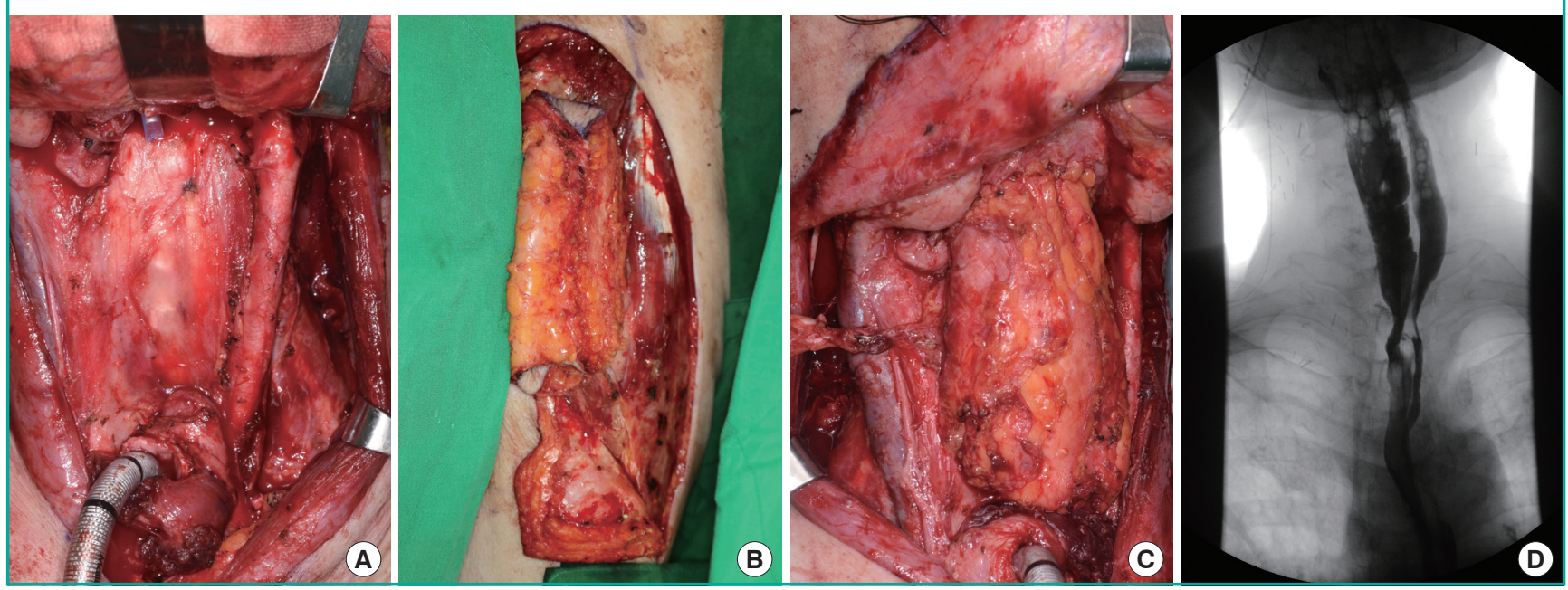
Table 2. Relationship between clinical factors and fistula incidence

\begin{tabular}{|c|c|c|}
\hline Clinical factor & Fistula & P-value \\
\hline Previous treatment & & $0.409^{\mathrm{a})}$ \\
\hline Primary tumor & $15(13.3)$ & \\
\hline Recurrent tumor & $3(21.4)$ & \\
\hline Tumor site & & $0.330^{\mathrm{a})}$ \\
\hline Oropharynx & $3(27.3)$ & \\
\hline Hypopharynx & $12(12)$ & \\
\hline Larynx & $3(18.8)$ & \\
\hline T stage & & $0.015^{b)}$ \\
\hline $\mathrm{T} 1$ & $0(0)$ & \\
\hline T2 & $3(6.5)$ & \\
\hline T3 & $3(10.7)$ & \\
\hline $\mathrm{T} 4$ & $12(24)$ & \\
\hline Type of flap & & $0.987^{\mathrm{a})}$ \\
\hline PMMC & $3(14.3)$ & \\
\hline Free flap & $15(14.2)$ & \\
\hline Type of free flap & & - \\
\hline RFFF & $15(15.5)$ & \\
\hline ALTFF & $0(0)$ & \\
\hline Type of reconstruction & & $0.007^{\mathrm{a})}$ \\
\hline Tube-type & $6(35.3)$ & \\
\hline Patch-type & $12(10.9)$ & \\
\hline Type of reconstruction (free flaps only) & & $0.006^{\mathrm{a})}$ \\
\hline Tube-type & $6(35.3)$ & \\
\hline Patch-type & $9(10.1)$ & \\
\hline Size of the flap & & $0.121^{\text {b) }}$ \\
\hline$<200 \mathrm{~cm}^{2}$ & $4(9.5)$ & \\
\hline $200-300 \mathrm{~cm}^{2}$ & $6(14.3)$ & \\
\hline $300-400 \mathrm{~cm}^{2}$ & $4(13.3)$ & \\
\hline $400-500 \mathrm{~cm}^{2}$ & $1(16.7)$ & \\
\hline$>500 \mathrm{~cm}^{2}$ & $3(42.9)$ & \\
\hline Previous radiotherapy & & $0.895^{\mathrm{a})}$ \\
\hline Yes & $16(14.0)$ & \\
\hline No & $2(15.4)$ & \\
\hline Preoperative albumin level & & $0.130^{\mathrm{a})}$ \\
\hline$\geq 3.5 \mathrm{~g} / \mathrm{dL}$ & $13(12.1)$ & \\
\hline$<3.5 \mathrm{~g} / \mathrm{dL}$ & $5(25)$ & \\
\hline Preoperative hemoglobin level & & $0.070^{\mathrm{a})}$ \\
\hline$\geq 12.5 \mathrm{~g} / \mathrm{dL}$ & $14(12.3)$ & \\
\hline$<12.5 \mathrm{~g} / \mathrm{dL}$ & $4(30.8)$ & \\
\hline Diabetes mellitus & & $0.116^{a)}$ \\
\hline Yes & $6(24)$ & \\
\hline No & $12(11.8)$ & \\
\hline Smoking & & $0.889^{\mathrm{a})}$ \\
\hline Yes & $1(12.5)$ & \\
\hline No & $17(14.3)$ & \\
\hline \multicolumn{3}{|c|}{ 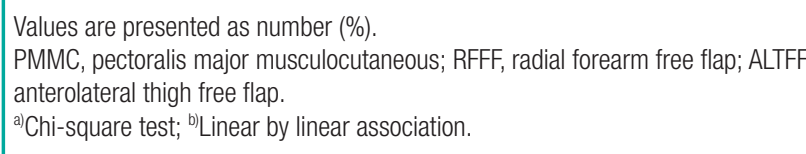 } \\
\hline
\end{tabular}

who underwent reconstruction with a patch-type radial forearm free flap was successfully treated with primary closure since the fistula was small, inflammation in the surrounding tissues was mild, and satisfactory bleeding was observed during debridement. In 4 cases with relatively clean wounds despite some tis- sue defects following debridement of the fistula and surrounding tissues, reconstruction with a PMMC flap was performed without pharyngostoma formation. In 3 of the 4 cases, the fistula recurred and was treated conservatively. In 11 cases in which the viability of the surrounding tissues following debridement was uncertain, a pharyngostoma was formed first. In 4 of these cases, direct closure was performed after the wound eventually stabilized, whereas in 2 of these cases where there was insufficient tissue, a pharyngostoma was formed via a PMMC flap and direct closure was performed after the wound eventually stabilized (Fig. 4). In another 4 cases, because of insufficient tissue during pharyngostoma closure, flap reconstruction was performed, with a PMMC flap in 2 cases, with a deltopectoral flap in 1 case, and with a local flap in 1 case. In 1 case, the patient died from multiple organ failure attributed to wound infection; excluding this case, in the 10 patients within the pharyngostoma formation group, the average duration until stoma closure was 45 days (range, $4-116$ days).

\section{DISCUSSION}

Despite the advancements in surgical techniques and the preventive efforts that have been described in numerous studies, the incidence of pharyngocutaneous fistula remains high, at $8 \%-22 \%$.

In the present study, higher $\mathrm{T}$ stages resulted in a significant increase in fistula incidence, which is consistent with the results of many previously reported studies $[5,10,11]$. Aarts et al. [10] reported that a high tumor stage was an independent prognostic factor of fistula incidence based on findings of incidence rates of $11 \%$ in tumors with an original stage of I and II and 35\% in tumors with an original stage of III and IV in patients who underwent salvage laryngectomy following primary radiotherapy. In contrast, several studies have reported no association between the $\mathrm{T}$ stage and fistula incidence $[1,3,7,12]$. Markou et al. [12] pointed out that any apparent association with patients who had an advanced $\mathrm{T}$ stage was due to errors from higher frequencies of infiltration at the excision margins.

Differences have also been reported in fistula incidence according to the type of flap used in reconstruction. Although the present study did not find a statistically significant difference between PMMC and free flaps, Chao et al. [14] reported that using a PMMC flap in partial defect reconstruction of the hypopharynx resulted in a significantly higher fistula rate than when a free fasciocutaneous flap was used $(24.7 \%$ vs. $8.9 \%, \mathrm{P}<0.0001)$; the reoperation rate due to fistula or flap necrosis was also higher, which was attributed to possible low peripheral vascularity caused by the arc of rotation of the PMMC flap or increased 


\section{Fig. 4. Pharyngostoma formation with a PMMC flap and direct closure}

A 51-year-old male with cancer of the hypopharynx. (A) A pharyngocutaneous fistula occurred on the upper suture margin after tube-type reconstruction. (B) A PMMC flap was transposed to make a pharyngostoma. (C) Pharyngostoma (postoperative day 41). (D) The upper third of the PMMC flap was turned over to make the pharyngeal wall. (E) The external skin wound was closed primarily. PMMC, pectoralis major musculocutaneous.
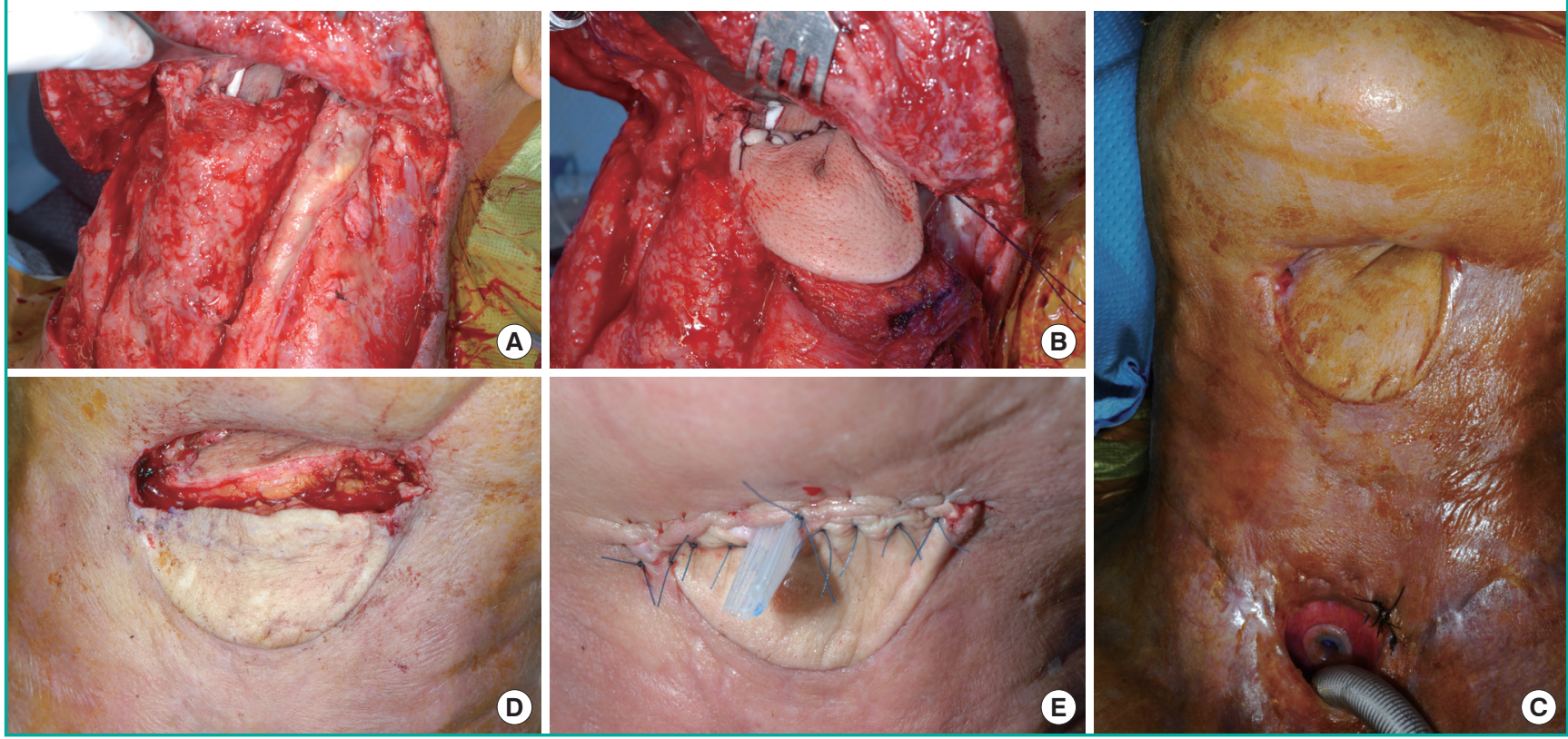

tension at the superior suture line caused by the downward weight of the bulky muscle. All 3 laryngeal tumor cases with a fistula in our study were stage $\mathrm{T} 4$ and the pharyngeal defect was reconstructed with a PMMC flap. We covered the suture line with the pectoralis major muscle to reinforce it, but a fistula occurred nonetheless. A radial forearm free flap is a thin and pliable flap that has consistent vascular pedicles, whereas an anterolateral thigh free flap is a wider flap that has a robust blood supply. Thus, they are both currently used for pharynx reconstruction. Kao et al. [13] reported that patients who underwent anterolateral thigh free flap reconstruction had a significantly lower fistula incidence than those who underwent radial forearm free flap reconstruction ( $56.6 \%$ vs. $30.2 \%, \mathrm{P}=0.03$ ), which was attributed to the anastomosis technique modification being more favorable because of the diverse and abundant tissues; for example, the surrounding fascia lata was harvested together to wrap the first-layer suture during flap elevation or the vastus lateralis muscle was included in the skin paddle. In the present study, although there was a difference in fistula incidence between the radial forearm free flap (15.5\%) and anterolateral thigh free flap (0\%) groups, the difference was not statistically significant.

Since in post-cancer resection cases, a remaining pharyngeal wall width of $<3 \mathrm{~cm}$ can result in impaired tissue perfusion when stretched, in the present study, the remaining tissue was removed and reconstruction with a tube-type flap was performed. Because a tube-type flap can become drooped by its own weight and a PMMC flap is pulled downward by the muscle pedicle, which can be exacerbated by scarring after subsequent radiotherapy, we fixed the flap to the prevertebral fascia with 3 or 4 sutures for tube-type reconstruction and PMMC flap reconstruction. In cases of a patch-type free flap reconstruction, we did not place such additional fixation sutures. In the comparison between tube- and patch-type reconstructions, the tube-type group showed a significantly higher fistula incidence when the PMMC flap reconstruction group was included in the patch-type group ( $35.3 \%$ vs. $10.9 \%, \mathrm{P}=0.007)$, as well as in the comparison with the free flap reconstruction group excluding PMMC flap reconstruction ( $35.3 \%$ vs. $10.1 \%, \mathrm{P}=0.006$ ). It is suggested that such results were observed because of the longer potential length for fistula formation from a longer suture line and the tension applied in multiple directions when a tube-type flap is used compared with a patch-type flap.

Salvage surgery for patients with recurrent or persistent tumors who have undergone radiotherapy is more prone to wound complications because of the presence of hypovascularized fibrotic tissue included in the radiation field. McCombe and Jones [8] reported that fistula incidence increased from $4 \%$ to $39 \%$ when radiotherapy was performed. Dirven et al. [9] reported an association between fistula incidence and the interval 
between radiotherapy and surgery; there was a distinct difference in the incidence rates in patients who underwent salvage surgery within 12 months of radiotherapy (48\%) and more than 12 months after radiotherapy ( $0 \%)$. In the present study, no statistically significant association was found between preoperative radiotherapy and fistula incidence, which may be attributable to the fact that the average interval between radiotherapy and surgery was 38 months (range, 2 months to 15 years), implying that surgery was performed when the surrounding tissues had already recovered to a certain degree.

Among patients with head and neck cancer, $30 \%-50 \%$ are in a malnourished state, which can worsen their prognosis and tolerance to antineoplastic therapy, while also lowering their metabolism and immune response; thus, there is an increased risk of infection that contributes to potential complications [15]. Albumin levels are a key indicator for assessing nutritional status, with numerous studies reporting that a low preoperative serum albumin level was associated with fistula incidence $[1,3,4]$. Moreover, anemia has also been reported to be associated with fistula incidence, since it impairs wound healing [4-6]. In the present study, albumin and hemoglobin levels were not significantly associated with fistula incidence; it is suggested that the association did not appear because the perioperative nutritional status, albumin level, and hemoglobin level were thoroughly controlled for in this study. In the present study, diabetes mellitus status likewise did not show a significant effect, which is consistent with the results of a study by Dedivitis et al. [5], in which reported comorbidities such as diabetes mellitus, chronic hepatopathy, hypothyroidism, and peripheral vascular disease did not affect fistula formation. In contrast, other studies have reported that hyperglycemia caused by insulin deficiency and insulin resistance exacerbated by both surgery and anesthesia caused impairments in wound healing and leukocyte function; thus, diabetes mellitus was strongly associated with fistula formation $[1,3]$. Therefore, controllable factors, such as perioperative nutritional status, albumin level, hemoglobin level, and blood sugar levels, should be strictly controlled both preoperatively and postoperatively.

It has been reported that fistula incidence was higher when the primary site of the cancer was the hypopharynx $[4,5]$ but the present study did not find any association between fistula incidence and the primary cancer site. Moreover, it has also been reported that fistula incidence was higher in supraglottic laryngeal cancer cases [5-7] but in the present study, such a comparison was impossible because of the small number of cases. We hypothesized that fistula incidence would be higher after salvage surgery because of vascular impairment and severe fibrosis from radiotherapy and previous operations in tissues within the oper- ating field, but no significant difference was observed. Moreover, it would seem that smoking could contribute to increased fistula incidence by delaying wound healing, but we did not find a significant association.

Once a fistula occurs, hospitalization can be prolonged and surgical treatment may be necessary, making the condition difficult to treat. Even if treated, it can result in sequelae such as stricture from scar formation; in general, flap reconstruction increases the potential for morbidity at the donor site. Therefore, it is important to prevent fistulae from occurring.

To prevent a fistula in patients who undergo flap reconstruction, it is necessary to have a sufficient blood supply and to eliminate any tension at the anastomosis site. The association between $\mathrm{T}$ stage and fistula incidence found in the present study can be linked to higher $\mathrm{T}$ stages requiring more extensive resection. Thus, the remaining tissue may be insufficient and cause tissue perfusion impairment or tension during anastomosis. The authors of the present study made it a general rule to remove any questionable tissue and to perform reconstruction with a sufficiently-sized flap to minimize tension at the suture line. Although it may appear that a larger flap size would result in a longer suture line, which would affect fistula incidence, the present study did not find any significant association between flap size and fistula incidence.

When insetting the flap, Soylu et al. [11] reported that switching the suture from chromic catgut to Vicryl reduced fistula incidence because Vicryl is much stronger, results in less inflammation, and has a longer half-life than catgut. In contrast, another study reported no difference between types of suture materials [7]. Moreover, Hamahata et al. [16] stated that fistula incidence was reduced by using a 3-layer suture method after circumferential de-epithelization of the skin at the flap margin during PMMC flap insetting, compared with a conventional 2-layer suture. In the present study, Vicryl 3-0 two-layer interrupted sutures were used in almost all cases; therefore, the effects of the suture material or suturing technique on fistula incidence could not be determined. Additionally, Chao et al. [14] used a method in which the suture line was reinforced by including an extra width of fascia during anterolateral thigh free flap harvesting, while Lopez et al. [17] stated that the fistula rate was lowered by reducing salivary exposure to the anastomotic suture line by stenting with a Montgomery salivary bypass tube during flap insetting. The authors of the present study did not use a salivary bypass tube during flap insetting in any cases.

With respect to treatments for fistula, improvement may be expected when the fistula is small, through wound packing and debridement at the bedside. A fistula can be treated by direct closure if discovered relatively early and if inflammation is mild. 
However, treatment with conservative management or direct closure becomes difficult when the fistula is discovered late because of poor vascularity in the surrounding tissue from chronic inflammation and infection, which may result in extensive necrosis. Even if such cases are treated, a stiff neck or difficulty in swallowing may develop due to the decreased lumen size in the affected area. In the present study, pharyngeal stricture occurred in 2 cases in which conservative management was applied, suggesting the need for more aggressive treatment from a long-term perspective.

Excluding cases with a small fistula and mild inflammation in the surrounding tissue, the authors of the present study made it a general rule to delay flap reconstruction until infection was controlled. We usually created a pharyngostoma first, after aggressive debridement of the fistula and surrounding tissue, to isolate the wound from the flow of saliva and pharyngeal contents. If the wound bed was clean and vascularity was clearly achieved after complete debridement, then immediate reconstruction using a flap also could be considered. However, in some cases the fistula recurred after a single-stage reconstruction without pharyngostoma formation. Therefore, rather than rebuilding immediately, we propose first making a pharyngosto$\mathrm{ma}$ and then considering closure or flap reconstruction once the wound becomes stable and clean (Fig. 5). It has been reported that negative-pressure wound therapy may be a useful alternative for patients who have contraindications to flap reconstruc- tion for fistula treatment [18]. However, the authors of the present study used negative-pressure wound therapy for cleansing purposes during dressing of infected wounds, but never during fistula treatment.

Pharyngocutaneous fistula requires global management from prevention to treatment. A precise preoperative evaluation is essential for prevention. Plastic surgeons should carefully observe a patient's performance status and general disease status, taking note of factors such as previous treatment history, laboratory data, and tumor status. The results of the present study show that a more careful approach is required for advanced-stage cancer or pharyngeal reconstruction with a tube-type flap; further, it is suggested that there is no need to set restrictions regarding using a flap large enough to minimize tension at the anastomosis site or flap selection appropriate for the patient's condition. Moreover, it is important to excise the remaining tissues suspected of vascularity after cancer resection as much as possible; to perform secure suturing in multiple layers during flap insetting; and to correct controllable factors such as nutritional status, albumin levels, hemoglobin levels, and diabetes mellitus by carefully evaluating the patient's condition during the perioperative period. It is believed that when treating fistulae, early detection and accurate assessment are the keys to successful treatment. Inflammatory changes should be identified as soon as possible and treatment should be initiated promptly to obtain better results and to reduce the probability of a large tissue de-

Fig. 5. Treatment algorithm for pharyngocutaneous fistula

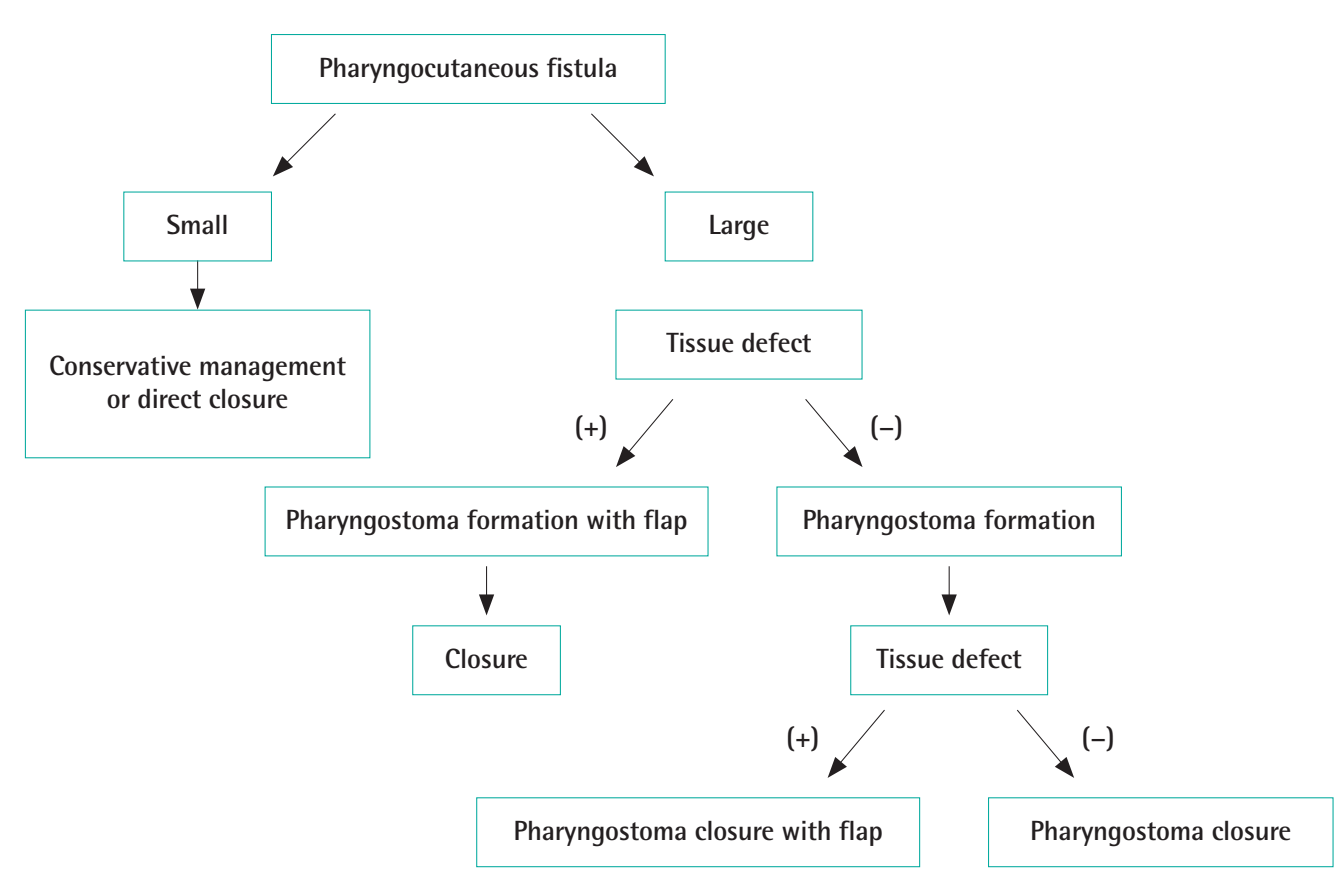


fect. Moreover, it is preferable to use a more cautious approach and to treat more aggressively by creating a pharyngostoma, in order to reduce the risk of treatment failure in these patients with few treatment options.

\section{CONFLICT OF INTEREST}

No potential conflict of interest relevant to this article was reported.

\section{PATIENT CONSENT}

The patient provided written informed consent for the publication and the use of their images.

\section{REFERENCES}

1. Mattioli F, Bettini M, Molteni G, et al. Analysis of risk factors for pharyngocutaneous fistula after total laryngectomy with particular focus on nutritional status. Acta Otorhinolaryngol Ital 2015;35:243-8.

2. Makitie AA, Irish J, Gullane PJ. Pharyngocutaneous fistula. Curr Opin Otolaryngol Head Neck Surg 2003;11:78-84.

3. Boscolo-Rizzo P, De Cillis G, Marchiori C, et al. Multivariate analysis of risk factors for pharyngocutaneous fistula after total laryngectomy. Eur Arch Otorhinolaryngol 2008; 265:929-36.

4. Leite AK, de Matos LL, Belli M, et al. Pectoralis major myocutaneous flap for head and neck reconstruction: risk factors for fistula formation. Acta Otorhinolaryngol Ital 2014;34: 389-93.

5. Dedivitis RA, Aires FT, Cernea CR, et al. Pharyngocutaneous fistula after total laryngectomy: systematic review of risk factors. Head Neck 2015;37:1691-7.

6. Benson EM, Hirata RM, Thompson CB, et al. Pharyngocutaneous fistula after total laryngectomy: a single-institution experience, 2001-2012. Am J Otolaryngol 2015;36:24-31.

7. Galli J, De Corso E, Volante M, et al. Postlaryngectomy pharyngocutaneous fistula: incidence, predisposing factors, and therapy. Otolaryngol Head Neck Surg 2005; 133:689-94.
8. McCombe AW, Jones AS. Radiotherapy and complications of laryngectomy. J Laryngol Otol 1993;107:130-2.

9. Dirven R, Swinson BD, Gao K, et al. The assessment of pharyngocutaneous fistula rate in patients treated primarily with definitive radiotherapy followed by salvage surgery of the larynx and hypopharynx. Laryngoscope 2009; 119: 1691-5.

10. Aarts MC, Rovers MM, Grau C, et al. Salvage laryngectomy after primary radiotherapy: what are prognostic factors for the development of pharyngocutaneous fistulae? Otolaryngol Head Neck Surg 2011;144:5-9.

11. Soylu L, Kiroglu M, Aydogan B, et al. Pharyngocutaneous fistula following laryngectomy. Head Neck 1998;20:22-5.

12. Markou KD, Vlachtsis KC, Nikolaou AC, et al. Incidence and predisposing factors of pharyngocutaneous fistula formation after total laryngectomy. Is there a relationship with tumor recurrence? Eur Arch Otorhinolaryngol 2004;261: 61-7.

13. Kao HK, Abdelrahman M, Chang KP, et al. Choice of flap affects fistula rate after salvage laryngopharyngectomy. Sci Rep 2015;5:9180.

14. Chao JW, Spector JA, Taylor EM, et al. Pectoralis major myocutaneous flap versus free fasciocutaneous flap for reconstruction of partial hypopharyngeal defects: what should we be doing? J Reconstr Microsurg 2015;31:198-204.

15. Brookes GB. Nutritional status: a prognostic indicator in head and neck cancer. Otolaryngol Head Neck Surg 1985; 93:69-74.

16. Hamahata A, Beppu T, Saitou T, et al. The usefulness of triple layers suturing technique with frilled pectoralis major musculocutaneous flap for pharyngocutaneous fistula. J Plast Reconstr Aesthet Surg 2014;67:e32-e33.

17. Lopez F, Obeso S, Camporro D, et al. Outcomes following pharyngolaryngectomy with fasciocutaneous free flap reconstruction and salivary bypass tube. Laryngoscope 2013; 123:591-6.

18. Loaec E, Vaillant PY, Bonne L, et al. Negative-pressure wound therapy for the treatment of pharyngocutaneous fistula. Eur Ann Otorhinolaryngol Head Neck Dis 2014;131: 351-5. 